\title{
The sleepwalking/night terrors syndrome in adults
}

\author{
AH Crisp
}

\section{Summary}

A third of a million adults in the UK sleepwalk while a million suffer from night terrors. In both conditions the individual is unaware of the fullness of their surroundings and is totally focussed in their concern or activity. Doctors are only likely to become involved if the individual comes to harm or seeks help or if other people are inconvenienced or threatened. The constitutional basis of the disorder is beyond doubt, although the actual expression may be related to stressful life-events resulting from an individual's personality, relationships and circumstances. Treatment may include the provision of a secure environment, counselling, and the use of benzodiazepines and serotonin re-uptake inhibitors.

Keywords: sleep disorders

\section{Sleepwalking/night terrors syndrome: epidemiology}

- sufferers may be totally unaware of the events

- a third of a million adults in the UK sleepwalk

- one million suffer 'night terrors'

- they are often 'closet' disorders

- expression of the syndrome often reflects an interaction between a constitutional propensity for the disorder (the abrupt escape of arousal into the brain when it is most dormant, and also the individual's personality) and stress

Box 1

Division of Psychological Medicine, Department of Mental Health Sciences, St George's Hospital Medical School, London, UK

AH Crisp

Correspondence to Professor AH Crisp, Psychiatric Research Unit, Atkinson Morley's Hospital, Copse Hill, Wimbledon, London SW20 ONE, UK

Accepted 3 January 1996
Somnambulism has long been recognised as an entity and has captured the public imagination over the centuries. Our poets and dramatists are convinced that, within it, the individual, inattentive to all but the task in hand, reveals feelings, wishes or impulses which are never expressed during full wakefulness and in the intact mental state. Moreover, such behaviour is presented as understandable in terms of a normally hidden aspect of that person's mind. Paradoxically, Shakespeare's Lady Macbeth expresses her guilt within this context; in wakefulness she is a demon! More often the picture painted is of the inherent goodness and compliance of the person when awake. Amina, in Bellini's ' $L a$ somnambula', can declare her love, albeit mistakenly, when sleepwalking - her well wishers are supportive in what they recognise as an expression of her true feelings; moreover she cannot be blamed since she is 'asleep'!

In everyday life, people who seemingly stumble upon unexpected outcomes or seem oblivious to the needs of others and impervious to outside influences are sometimes loosely described as 'sleepwalking'. Such attributions have embraced both the righteous (eg, Koestler's 'sleepwalkers') and the unholy (eg, Adolf Hitler) and emphasise the possibility of similar sustained mechanisms within the personality that can operate independently of sleep. However, there are no clinical links with the classical sleepwalking syndrome and such behaviours, if pathological, are more usefully contained diagnostically within categories of personality and its disorders.

It is natural to 'habituate' to aspects of the environment that have become insignificant for us and to those of our actions that have become 'automatic' (eg, when driving our car along an established route we may barely notice many elements of the journey). This inattention differs, at least spectrally, from the potential for disconnecting, as a mentally defensive strategy, from seriously distressing states or environments. This protective restriction of the sensorium has been labelled 'dissociation' and may be harnessing an important and primitive neurophysiological mechanism subserving emotional/arousal homeostatic needs, when other more sophisticated defences fail. In part, the sleepwalking and night terror syndrome was for a long time confidently identified as such a dissociative disorder but arising uniquely out of sleep.

More recently, night terrors (incubus attacks) have also been identified as an entity, distinct from nightmares, and as first cousins to somnambulism. The overall syndrome fits comfortably as an high profile entity within the 'parasomnias' group of sleep disorders with characteristic polysomnographic status (F 51.3 and F 51.4, ICD10). ${ }^{1}$ Within both variants of the syndrome, the behaviour classically erupts out of deep (stage 3/4) non-rapid eye movement sleep (non-REM) in the first or second sleep cycle. Figures 1 and 2 show, respectively, a normal hypnogram and one characteristic of the syndrome. Sleepwalking may be the only feature of the syndrome, as may a night terror. Alternatively, events that start as night terrors may spill over into sleepwalking.

In both conditions the individual is unaware of the fullness of their surroundings and is totally focused on their concern or activity. The movements may be rather clumsy and only semi-purposeful but can be forceful. Return to sleep can occur without full awakening and then there will be no memory of the event the next day. If waking does occur it can be prolonged but not necessarily so. Some habitual adult sleepwalkers are only aware that walking has occurred because they subsequently find that objects in their room are rearranged, etc.

Traditionally, night terrors are said to be relatively contentless. The individual wakens with a start, may scream or gasp and looks terrified. In contrast, nightmares more commonly erupt later in the night and out of REM sleep. Nightmares are considered to be frightening dreams that waken the individual and are characterised by vivid mental content. However, some of these distinctions may not be so absolute, and classical night terrors (eg, 
Figure 1 Normal sleep hypnogram in a young adult showing minimal sleep interruptions and well-organised rhythmic activity and with the bulk of slow wave sleep (stages 3 and 4) being experienced within the first two hours. Key: MA: minor arousal; MT: movement time

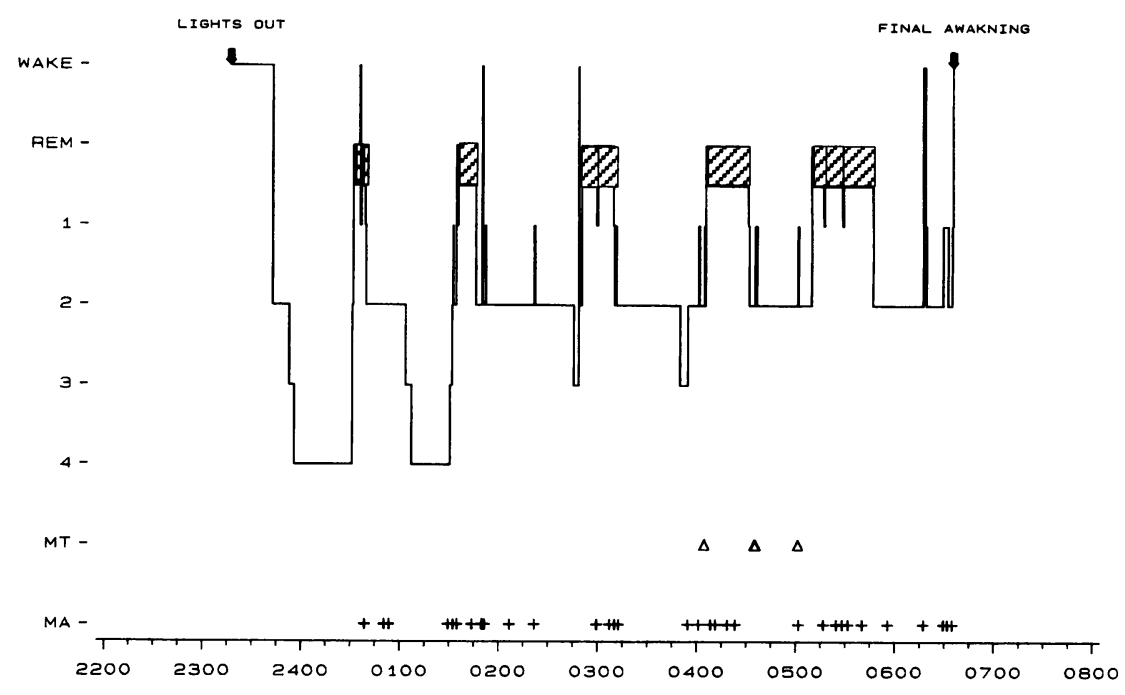

Figure 2 Classical hypnogram of patient with the sleep walking/night terrors syndrome. On this particular night there was no clinical event but the tracing still shows half-a-dozen abrupt stage 4-0 transitions (arousals) which characterise the syndrome and out of which sleepwalking/night terrors nearly always erupt. Also evident was the substantial amount of stage 4 sleep later in the night which is probably a product of earlier slow wave sleep deprivation due to the repeated arousals. Key: MA: minor arousal; MT: movement time; $\nabla$ : stage 4-0 transitions

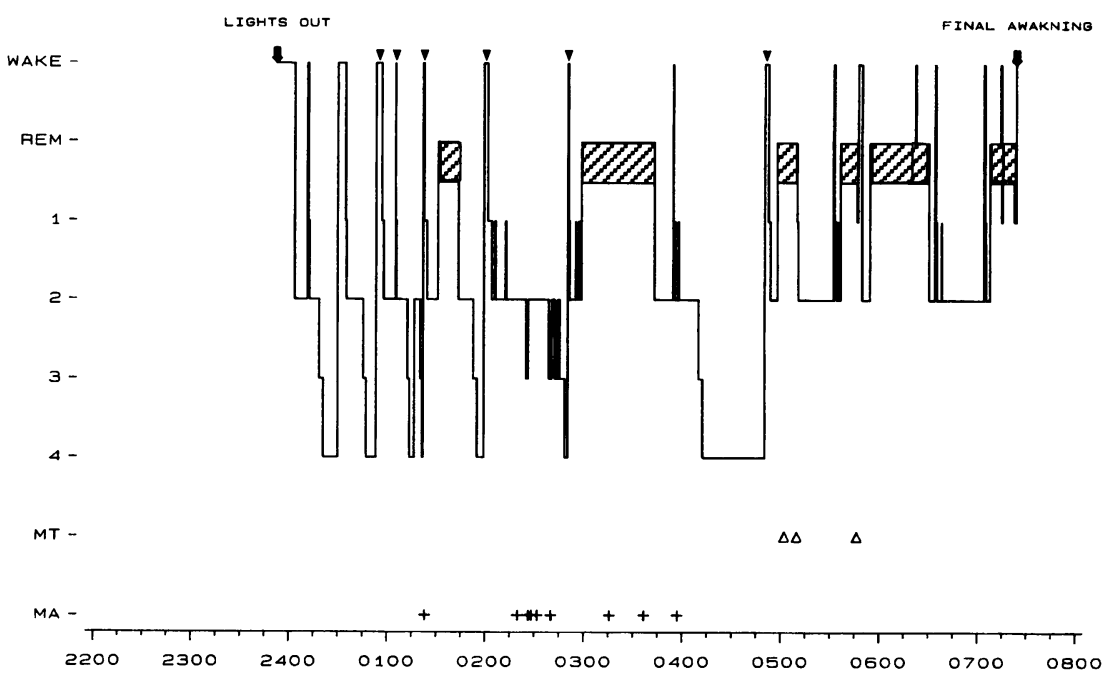

erupting unequivocally from deep non-REM sleep within an hour or two of going off to sleep, with or without subsequent sleepwalking) can also have vivid content. So long as awareness remains restricted the individual remains totally identified with his or her frightening experience and cannot be reassured.

\section{Epidemiology}

The overall syndrome is common in early life and one or more events are reported by or observed in about $25 \%$ of children/young adolescents. Within clinical populations it is said to be more common in good, compliant, children who do not show their emotions. ${ }^{2}$ A typical view is that it is an expression of the immature brain but clearly more acute emotional and social factors also operate and can generate or protect against behavioural expression of the syndrome. Classified as a 'parasomnia' it sits alongside other conditions such as sleeprelated enuresis, another syndrome common in childhood and out of which people are expected to 'grow'.

Less is heard of the syndrome in the adult. Most often it has then prevailed since childhood and advice and more specific treatment has not worked. Such individuals learn to live with their condition. Persistent nocturnal enuresis often pursues this same course. It is clear that $1-2 \%$ of adults report somnambu$\operatorname{lism}^{3} ; 4-5 \%$ continue to experience night terrors. The disorder is about twice as common in adult males compared to females. Occasionally adults can develop the syndrome for the first time. Such events may occur every night or even several times per night. Associated with other frequent awakenings within sleep, they can leave the individual exhausted and often perplexed the next day. In other cases the disorder is episodic. Most such people live out their lives 


\begin{tabular}{|l|}
\hline Complications \\
\hline - exhaustion \\
- self-injury \\
- injury to others \\
\hline
\end{tabular}

Box 3 without seeking further medical advice. Doctors are only likely to become involved if:

- the individual comes to harm, eg, falls down the stairs

- other people are inconvenienced or threatened

- the individual seeks help because of endless sleep interruptions (which they recognise and remember if they have ultimately wakened after events) and/or related exhaustion

- secondary complications such as alcohol dependence arise (in such cases the patient has most often resorted to alcohol in an attempt to block awakenings in the first four hours of the sleep period and after prescribed hypnotics have failed to bring any such relief).

\section{Sleep and wakefulness}

The very name 'sleepwalking' preempts the discussion - is the individual asleep or awake? Clearly he or she is neither fully awake nor typically asleep. Early clinical studies came mainly from psychiatry and emphasised the operation of mental 'dissociative' mechanisms, akin to those claimed to be operating in the generation of hysterical symptoms. ${ }^{4}$ The syndrome of hysterical amnesia is perhaps the closest parallel. These views were in accord with the vision of the dramatists - some aspect of the individual's mind, eg, their capacity for denial of distress or their self-critical faculty, was suspended; resistance to expression of fear, wish or impulse was lost.

At a more simple observational level, Maria De Maraceine, an outstanding physiologist writing at the turn of the century, reported her observation ${ }^{5}$ that children, wakened from deep sleep an hour after going to bed, were often difficult to arouse fully; they would adopt a trance-like state the duration of which, she concluded, was proportional to their degree of 'immaturity' and 'nervousness'. Returning once again to that other comparable parasomnia, many parents today discover the same phenomenon when attempting to waken their potentially enuretic child. Although the child accompanies them to the toilet, waking is never complete and subsequent bedwetting is often not averted.

The early psychodynamic concepts concerning sleepwalking, which implied a restricted sensorium generated by this mental defence mechanism of dissociation, came under further critical scrutiny 30 or 40 years ago on several accounts. Firstly, it is less plausible as a mechanism that might account for night terrors. Dissociation is perceived as protecting against anxiety, not unleashing it, albeit within a state of restricted awareness. Secondly, for a while, the related syndrome of 'hysteria', itself thought to have probably been rooted in a mental dissociative mechanism, came instead to be regarded as a prodromal feature of underlying organic cerebral pathology (eg, a tumour) likely to declare itself in grosser form at a later stage. This is now recognised to be rarely the case. Thirdly, however, the advent of polysomnography revealed electrophysiological characteristics of the syndrome (figures 2 and 3), which some people immediately concluded reflected its only true constitutional basis, inviting a physiochemical approach to treatment. Indeed it became clear that nearly all such events erupted suddenly out of slow wave sleep. ${ }^{6,7}$ However, it is noteworthy that both these research teams also emphasised the psychopathological aspects of the disorder. Fisher and his colleagues ${ }^{6}$ suggested that they arose because of a breach in the subject's ability to control anxiety.

Meanwhile, Broughton ${ }^{8}$ emphasised the sudden intense arousal characteristics of the disorder. Debate continued as to whether there was initial persistence of slow wave cerebral activity within the arousal. Indeed, close study of figure 3 suggests that there is. Some people took this as evidence that the individual was still 'asleep'. However, such rhythmic activity has been reported

Table Presentations that commonly distinguish between sleepwalking, night terrors and nightmares

\begin{tabular}{|c|c|c|c|}
\hline & Sleepwalking & Night terrors & Nightmares \\
\hline $\begin{array}{l}\text { Apparent/reported personal } \\
\text { experience }\end{array}$ & singleminded concern & fear, defencelessness & fear, defencelessness \\
\hline Mental content & singleminded concern & sometimes contentless & vivid/dreamlike \\
\hline Awareness on arousal & restricted & restricted & full \\
\hline Behaviour & $\begin{array}{l}\text { purposeful, clumsy, sterotyped } \\
\text { for individual }\end{array}$ & cowering & $\begin{array}{l}\text { rapid shift to wakefulness; } \\
\text { conscious movement thereafter }\end{array}$ \\
\hline Timing within sleep & usually first hour or so & usually first hour or so & later in night \\
\hline $\begin{array}{l}\text { Preceding polysomnographic } \\
\text { sleep stage }\end{array}$ & 4 & 4 & REM sleep \\
\hline Most likely personality & striving & striving/anxious & dysphoric \\
\hline
\end{tabular}


by others as also being a general feature of aspects of wakefulness, ${ }^{9}$ especially in the periods surrounding sleep (and perhaps associated with relative inattention to the environment) and also as characterising wakefulness in the emotionally 'immature' adult. This intriguing debate continues to this day. At our present level of medical understanding it is perhaps a semantic issue as to whether the individual is awake or asleep. They appear, to this writer, to be partially awake but not sensorially intact. Such concern has to be brought to bear when, rarely, forensic problems arise (eg, frightening assault on a bed partner or even homicide). Opinions vary from those who argue that the given behaviour is completely alien to the individual when fully awake and also that it is clearly occurring within a restricted degree of wakefulness such that the individual cannot be held responsible for the action, through to those who claim that the behaviour can represent a long-hidden wish or impulse for which the individual must accept some responsibility. The problem is complicated further by the obvious possibility that some individuals might simulate the syndrome. This has almost certainly occurred and, classically, presents with much more in the way of of elaborate and dramatic accompaniments. Meanwhile, it is the context of $\vec{a}$ sleep that protects the genuine sleepwalker under these circumstances from otherwise attracting a diagnostic label such as multiple personality disorder ( $\mathrm{F}$ 44.81, ICD $10^{1}$ ).

Broughton and his colleagues ${ }^{10}$ have recently produced an admirable report on the medicolegal complexities of 'homicidal somnambulism', including careful consideration of the occasional difficulties in diagnosis and the need to differentiate the condition from partial seizures, REM sleep behaviour disorder, sleep drunkenness, and dissociative and volitional behaviours within underlying wakefulness.

The present author and his colleagues ${ }^{11}$ have searched psychometrically for personality correlates of polysomnographically confirmed somnambulism and found an unusual psychological profile of normality, apart from high hysteria scores. Patients with night terrors presented similarly, but also had high anxiety scores. The questions within the hysteria scale most often responded to positively were those to do with enjoying acting, dramatic situations, being the centre of attention, prone to posing and pretending and, also, in the case of night terrors, being excessively emotional. Remarkably, few of these patients displayed such behaviour within wakefulness although they reported it in their questionnaire answers, suggesting that they were aware of an inner self not expressed in their normal wakefulness. The finding of such apparent 'normality' within wakefulness, albeit in a clinic population of sleepwalking/night terrors patients, resonates with the Klackenberg finding in children referred to earlier. ${ }^{2}$ It suggests a constitutional basis to the disorder reflected physiologically, behaviourally, and experientially. Such an escape of feelings (as suggested by Fisher) and behaviour within slow wave sleep when blood supply to the cerebral cortex is minimal and the mind's more cerebrocortically shaped defences are at rest, would fit with this profile. Such a personality structure might also require, under these circumstances, that the normal wakeful/conscious self remains dormant via a more massive cerebral dissociative process. However, it remains to be seen whether these personality features characterise all sleepwalkers; it could be argued that they reflect the impact of the chronic disorder on the individual.

Meanwhile, the polysomnography of people with sleepwalking is not only characterised by sudden arousal from stage 3/4 sleep, which might or might not spill over into an episode of night terrors/sleepwalking; such sudden arousals, perhaps never extending to full consciousness and not remembered the next morning, can not only occur asymptomatically several times within deep sleep but can also, to a lesser extent, characterise much of the remainder of sleep. However, sleepwalking/night terror 'events' rarely occur from these latter arousals.

Moreover, patients with this syndrome usually demonstrate a chronic inability to stay in deep sleep. The moment they enter it, so to speak, they are vulnerable to sudden arousals. This disturbance of their slow wave sleep and the slow wave $\mathbb{\Phi}$ sleep debt which they may thereby build up, probably accounts for the continued : reappearance of slow wave sleep later in the night and at a time when it otherwise occurs less characteristically. Thus it is common to see slow wave sleep appearing within all the sleep cycles. When slow wave sleep is thereby substantially driven into the second half of the night, it remains prone to sudden arousals but which less often lead to events. Patients with the syndrome sometimes report tiredness the next day when such arousals are frequent, especially if they have been accompanied by one or more episodes of night terrors/sleepwalking. Attempts at self-medication with alcohol may complicate the picture.

The constitutional basis for the disorder is beyond doubt. Apart from the above characteristics, its chronic expression and tendency to run in families and reported high concordance in identical twins all lend support to this 


\section{Dramatists over the centuries have recognised the essence of the syndrome}

Extract from Macbeth, Act V, Scene 1

In this scene Shakespeare allows a gentlewoman to instruct the doctor in the features of the syndrome.

Doctor: I have two nights watched with you, but can perceive no truth in your report. When was it she last walked?

Gentlewoman: I have seen her rise from her bed, throw her nightgown upon her, unlock her closet, take forth a paper, fold it, write upon't, read it, afterwards seal it, and again return to bed; yet all this while in a most fast sleep.

Doctor: A great perturbation in nature! To receive at once the benefit of sleep, and do the effects of watching. In this slumbery agitation, besides her walking and other actual

performances, what, at any time have you heard her say?

Gentlewoman: That, sir, which I will not report after her. . . . Lo, you, here she comes! And, upon my life, fast asleep. Observe her: stand close.

Doctor: You see her eyes are open. Gentlewoman: Ay, but their sense is shut.

Doctor: . . . Look how she rubs her hands.

Gentlewoman: . . . I have known her continue in this a quarter of an hour. . Lady Macbeth: Out, damned spot! Out I say . .

Doctor: This disease is beyond my practice: yet I have known those which have walked in their sleep who had died holily in their beds.

Bellini's opera La Somnambula (text by Felice Romani) reveals a similar vision of sleepwalking, though this time apparently the specific nature of the event in an innocent maiden is driven by chance. After regaining wakefulness, Amina's protestations of innocence are sincere though not believed by her fiancé.

She sings beseechingly:

"D'un pensiero, e d'un accento"

"Not in (my) thoughts' remotest regions".

\section{Box 4}

\section{Treatment}

- pharmacological, eg, temazepam $10-30 \mathrm{mg}$ before retiring, paroxetine (Seroxat) $20 \mathrm{mg}$ evening dose (worth trying)

- behavioural, eg, protective environment

- psychological proposition. ${ }^{12}$ However, the earlier notion of the role of 'dissociation', as a mental mechanism accounting for the condition, deserves further attention. Firstly, as previously mentioned, as a propensity it is thought to be a constitutional feature of some individuals, a brain function related to other aspects of personality. As also previously touched upon, such mental states are convincingly present in some people within wakefulness - 'hysterical amnesia' being perhaps the most classical. The mechanism has also been implicated in multiple personality disorder. The sudden arousal from deep sleep characterising the syndrome and normally contained within 'watchful' wakefulness might reflect part of this mechanism or the mechanism may simply operate in the predisposed in response to such arousal. Thus, most of us, wakened from deep sleep, experience initial disorientation and struggle successfully to orientate ourselves. Most doctors, for instance, on call and wakened from deep sleep by a telephone ringing after having themselves been asleep for just an hour or two, will remember the experience well. If a sufficiently established dissociative tendency is present, it might come into operation at that point, blocking full awareness and allowing internal and substantially buried psychological concerns to hold sway. Perhaps this mechanism and the possible related sudden escape of arousal into the brain is best looked upon as a universal mechanism, like the potential for epileptic discharge, but expressing itself clinically in just a small proportion of the adult population under normal circumstances.

The behaviour of sleepwalkers is usually much less dramatic or apparently meaningful than the nonmedical literature would have us believe, though it is often reported on by patients and bed partners as being more elaborate than is evident within the alien and perhaps muting environment of the sleep laboratory. Nevertheless it can still sometimes give the impression of being a vestigial expression of a psychological conflict, an impulse or a reaction, for instance, to loss (eg, searching behaviour) importantly related to an earlier phase of the individual's life. Detailed psychological exploration at this level can draw on such tools as personal construct theory and related repertory grid techniques, ${ }^{13}$ which might prove useful in trying to explore the meaning of such behaviour when it offers a clinical clue to its significance.

\section{Treatment}

The need to try to help such individuals remains paramount for doctors. Ordinary careful clinical enquiry of a biographical, life-event and mental state kind will often reveal links between the recrudescence of sleepwalking/night terrors, life-events and related strains stemming from the individual's personality, relationships and circumstances. This psychosomatic model, ie, a constitutional propensity, moderated by degrees of stress and with expression of the disorder thereby either muted or else exposed, invites attention to both aspects. Mechanical approaches include the provision of a secure environment and the deployment of certain drugs that have demonstrable effect. Drugs that either especially intensify slow wave activity, (eg, central nervous system (CNS) depressants) or else block it (eg, CNS stimulants) are of no value and should be avoided. Benzodiazepines can be helpful in reducing the severity of symptoms and have their staunch advocates. ${ }^{14,15}$ More recently there has been a case report claiming great benefit from a selective serotonin re-uptake inhibitor. ${ }^{16}$ The authors emphasise the specific effects that this group of drugs has on slow wave sleep in particular. Their possible benefit deserves further attention. Such drugs may also operate by modifying levels of underlying dysphoria which otherwise prompt the sudden arousals from deep sleep, and may be especially helpful when a night terror component to the syndrome is to the fore. All such drugs need to be used with discretion, given the chronic nature of the condition and the frequent presence of underlying psychological strain. Counselling approaches themselves need to address any psychological strains that have been identified as clinically relevant, eg, in terms of personal development, personality, current relationships and related life-events and sometimes reflected in the mental content of the syndrome. Such approaches, if expert, can sometimes alter the pattern, at least temporarily, even if sleepwalking/night terrors has been the nightly norm for many years.

These syndromes have been neglected by health carers. They can be seriously incapacitating. A greater understanding of them might shed further light, not only on the nature of sleep but also on mechanisms of the mind.

The author wishes to acknowledge the help of Ms Sharon Borrow for the generation of the relevant polysomnographic data and Mr Philip Sedgwick for its construction in hypnographic form (figures 1 and 2). 
1 World Health Organisation (WHO). The ICD10 classification of mental and behavioural disorders. Geneva: WHO, 1992.

2 Klackenburg G. Somnambulism in childhood prevalence, course and behavioural correlations. Acta Paediatr Scand 1982; 71: 495-9.

3 Bixler EO, Kales A, Soldatos CR, Kales JD, Healey S. Prevalence of sleep disorders in the Los Angeles metropolitan area. Am $\mathcal{f}$ Psychiatry 1979; 136: $1257-62$.

4 Sours JA, Frumkin $P$, Indermill $R$. Somnambulism. Arch Gen Psychiatry 1963; 9: $400-13$.

5 De Maraceine M. Sleep: its physiology, pathology, hygiene and psychology London: Walter Scott, 1897.

6 Fisher C, Kahn E, Edwards A, et al. A psychophysiological study of nightmares and night terrors. I. Physiological aspects of the stage 4 night terror. $\mathcal{F}$ Nerv Ment Dis 1973; 157: 75 98.
7 Kales A, Jacobson A, Paulson M, et al. Som nambulism: psychophysiological correlates. All night EEG studies. Arch Gen Psychiatry 1966; 14: 586-94.

8 Broughton $\mathbf{R}$. Sleep disorders and disorders of arousal. Science 1968; 159: 1070-8.

9 Brunner DP, Dijk D-J, Borbély AA. Repeated partial sleep deprivation progressively changes the EEG during sleep and wakefulness. Sleep 1993; 16: $100-13$.

10 Broughton R, Billings R, Cartwright $\mathrm{R}$, et al. Broughton $\mathbf{R}$, Billings $\mathbf{R}$, Cartwright $\mathbf{R}$, et al. 1994; 17: $253-64$

11 Crisp AH, Matthews BM, Oakey M, Crutchfield $M$. Sleepwalking, night terrors, and consciousness. BMf 1990; 300: 360-2.

12 Kales A, Soldatos CR, Bixler EO, et al. Hereditary factors in sleepwalking and nigh terrors. Br f Psychiatry 1988; 137: $111-8$.

13 Kelly GA. The psychology of personal constructs Vols I and II. New York: Norton, 1955.
14 Schenck CH, Milner DM, Hurwitz TD, Bundlie AR, Mahowald MW. A polysomnographic and clinical report on sleep-related injury in 100 adult patients. Am f Psychiatry 1989; 146: $1166-73$.

15 Kavey NB, Jamie Whyte AB, Resor SR, GidroFrank S. Somnambulism in adults. Neurology 1990; 40: 749-52.

16 Lillywhite AR, Wilson SJ, Nutt DJ. Successful treatment of night terrors and somnambulism with paroxetine. Br f Psychiatry 1994; 164: 551 -

\section{Medical Anniversary}

\section{AXEL MUNTHE, 31 OCTOBER 1857}

Axel Martin Fredrik Munthe (1857-1949) was born at Oskarshamm, Sweden, the youngest of three children of a prosperous professional family. He qualified in Paris (1879) with a thesis on uterine haemorrhage. He practised medicine in Capri, Paris, Rome, Stockholm and also had the time to write The story of San Michele (1929), perhaps the most popular medical autobiography ever. He married a wealthy English girl, Hilda Pennington in 1907 and lived at 31 St James' Place, Piccadilly, London, where two sons were born. He became physician to Crown Princess Victoria of Sweden in 1908 and was a close friend of King Gustav of Sweden. He died of pneumonia on 11 February 1949. His dream home, San Michele in Capri, was left to Sweden to house the Swedish Institute of Art. - DG fames 